\title{
GOUT AND ITS EFFECT ON THE CARDIOVASCULAR SYSTEM *
}

BY

\section{G. LEROY STEINBERG}

The characteristic feature that distinguishes gout from the other syndromes of rheumatic disease is an abnormality of uric acid metabolism. Uric acid exists in the body both as free acid and as the more soluble monosodium salt. Approximately half is endogenous in origin and the remainder is derived from digestion of nucleoproteins and nucleic acid in the food. The total amount of urate excreted in the urine is normally about $0.75-1.00$ gramme per day, and the quantity is increased by ingestion of any type of protein and decreased by a high fat dieta mechanism utilized by Lockie and Hubbard (1935) and Lockie (1942) in order to test for gout by attempting to promote an acute attack in a suspected case by placing him on a ketogenic diet.

Since the time of Thomas Sydenham (1683), himself a sufferer, gout has been blamed as one of the causes, or at least a concomitant, of kidney damage and renal calculus. The frequent occurrence of hypertension and vascular lesions in gout is recorded by many authorities (Meakin 1939, Osler and McCrae 1925, Cecil 1943, Levine 1940 and Stotzer 1939), while Garcin (1939) gives a detailed description of the histology of the gouty kidney. Only Rosenbloom (1918) and Buckley $(1937,1938)$ state that this association is not constant, and often absent.

Master and others (1943) have estimated that the incidence of hypertension in persons over 40 years of age in the general population is not more than 50 per cent. The term hypertension in their report denotes (1) a systolic pressure of $150 \mathrm{~mm}$. $\mathrm{Hg}$ or more at any time during observation, (2) a diastolic pressure of $96 \mathrm{~mm}$. or more prior to an attack of coronary occlusion, (3) a diastolic pressure of $90 \mathrm{~mm}$. or more during or after the attack, (4) marked enlargement of the heart without obvious cause. The application of these criteria shows that 69 per cent. of the patients afflicted with coronary occlusion had hypertension before the attack. The incidence in the general population, including men, women, and children, is much less than Master's figures. During the past ten years, 83,514 patients were admitted to one of our large local general hospitals. During a similar period 10,073 patients with either coronary insufficiency or coronary occlusion, and 1,819 patients with essential hypertension (with no demonstrable coronary disease), were admitted.

* This is an abridgment of an articie submitted for publication.
Forty-six cases of typical gout are included in this study. All the patients have been observed and treated by the writer, and most of them have been observed over a period of several years. The age of a patient listed in Table 1 is the age at which the patient was first seen. Thirty-one patients had a normal blood pressure (Table 2) and 15 had hypertension (Table 3). Only 2 patients of the hypertensive group were under 45 years of age. If one assumes that the average percentage of hypertension occurring in adults past the age of 45 is 50 , then hypertension among the gouty is no higher than in the average population. However, a more convincing argument against the view that gout produces hypertension is a study of the duration of the disease and its effect on the blood pressure. Eighteen cases of gout in the non-hypertensive group had their gouty symptoms for a period of five years or over; 13 cases had clinical gout for a period of ten years or over, 9 cases for a period of fifteen or more years, and 4 for a period of at least twenty years. Most impressive is the fact that Case 13, that of a white male aged 72 , had a history of recurrent attacks of gout for a period of forty years and still had a normal blood pressure. No cardiac enlargement and no symptoms of angina were present in the nonhypertensive group. Two patients developed hypertension while being observed. The blood pressure in one case rose from $140 / 100$ to $190 / 110$ during a period of four years, and the blood pressure in the other case rose from $140 / 80$ to $160 / 106$ during a period of two years' observation. The first case had gout for a period of nine years, and the second for twenty years.

Nine cases of gout revealed a trace to a $1+$ albumin in the urine at different periods of observation. Three of these nine cases also had hypertension. The ages of these patients varied from 45 to 62 years. Only one patient of the entire group of 46 cases had a renal calculus, which was present seven years before the onset of the clinical gout. The ability of the kidney to concentrate was good in all 46 cases, as demonstrated by a urine with a specific gravity of 1020 or higher.

Symptoms of angina were present in three cases: Two of these patients had hypertension. One patient aged 39 years had had gout for five years, 
TABLE 1

A Clinical Interpretation of Gout

\begin{tabular}{|c|c|c|c|c|c|c|c|c|}
\hline Case & Sex & Age & Duration & Blood pressure & Heart & E.C.G. & Weight & Urine \\
\hline $\begin{array}{l}1 \\
2\end{array}$ & $\begin{array}{l}\mathbf{M} \\
\mathbf{M}\end{array}$ & $\begin{array}{l}57 \\
60\end{array}$ & 10 years & $140 / 80$ & Negative. & & $150 / 140$ & Negative. \\
\hline 3 & $\mathbf{M}$ & 51 & $24 \quad \ddot{r}$ & $124 / 90$ & $\begin{array}{l}\text { Systolic and di- } \\
\text { astolic. base and } \\
\text { apex. }\end{array}$ & & $177 / 184$ & $\begin{array}{l}\text { Trace albumin; oc- } \\
\text { casional hyalin } \\
\text { cast. }\end{array}$ \\
\hline 4 & $M$ & 47 & 7 months & $140 / 110$ & Negative & & $224 / 198$ & Negative. \\
\hline $\begin{array}{l}5 \\
6\end{array}$ & $\begin{array}{l}\mathbf{M} \\
\mathbf{M}\end{array}$ & $\begin{array}{l}39 \\
53\end{array}$ & 3 years & $\begin{array}{l}140 / 100 \\
120 / 80\end{array}$ &. & & $170 / 144$ & $\cdot$ \\
\hline$\stackrel{6}{7}$ & $\mathbf{M}$ & $\begin{array}{l}53 \\
68\end{array}$ & $\begin{array}{rr}7 & . . \\
20 & . .\end{array}$ & $\begin{array}{l}120 / 80 \\
120 / 80\end{array}$ & $\ddot{.}$ & & & Faint trace albumin \\
\hline 8 & $M$ & 56 & $10 \quad \because$. & $114 / 80$ & $\ddot{.}$ & & & Negative. \\
\hline 9 & M & 42 & $4 \quad \cdots$ & $120 / 80$ & ‥ & & $202 / 194$ &.. \\
\hline 10 & $\mathbf{M}$ & 51 & $5 \quad .$. & $120 / 80$ & $\begin{array}{l}\text { Systolic, apex and } \\
\text { base. }\end{array}$ & & $188 / 157$ & . \\
\hline 11 & M & 61 & $10 \quad$. & $180 / 100$ & $\begin{array}{l}\text { Systolic. praecor- } \\
\text { dium. }\end{array}$ & & $178 / 142$ & . \\
\hline 12 & M & 41 & $7 \quad$. & $136 / 90$ & Negative. & & $207 / 174$ & $\begin{array}{l}\text { Albumin, Oc- } \\
\text { casional granular } \\
\text { cast. }\end{array}$ \\
\hline $\begin{array}{l}13 \\
14\end{array}$ & $\begin{array}{l}\mathbf{M} \\
\mathbf{F}\end{array}$ & $\begin{array}{l}45 \\
53\end{array}$ & 2 weeks & $\begin{array}{l}140 / 80 \\
170 / 100\end{array}$ & $\ddot{\prime}$ & & $\begin{array}{l}175 / 151 \\
165 / 148\end{array}$ & Negative. \\
\hline & & & & $\begin{array}{l}\text { (Cerebral haemor- } \\
\text { rhage } 4 \text { years } \\
\text { later.) }\end{array}$ & , , & Negative. & $165 / 148$ & D. \\
\hline $\begin{array}{l}15 \\
16\end{array}$ & $\begin{array}{l}\mathrm{M} \\
\mathrm{M}\end{array}$ & $\begin{array}{l}39 \\
40\end{array}$ & $\begin{array}{l}13 \text { years } \\
14 \quad,\end{array}$ & $\begin{array}{l}120 / 72 \\
120 / 80\end{array}$ & $\ddot{~, ~}$ & $\because$ & 250 & $\begin{array}{l}\text { Rare hyaline cast. } \\
\text { Negative. }\end{array}$ \\
\hline 17 & M & 45 & $4 \quad \because$ & $120 / 80$ & . & $\cdots$ & $175 / 153$ & \\
\hline $\begin{array}{l}18 \\
19\end{array}$ & $\mathbf{M}$ & 55 & 4 months & $\begin{array}{r}120 / 80 \\
140 / 90\end{array}$ & $\because$ & & $165 / 149$ & Trace albumin. \\
\hline 19 & & 47 & 9 years & $\begin{array}{l}140 / 90 \text { and four } \\
\text { years later } 190 / \\
110 \text {. }\end{array}$ & & ". & $183 / 163$ & Albumin, - : \\
\hline $\begin{array}{l}20 \\
21\end{array}$ & $\begin{array}{l}\mathrm{M} \\
\mathrm{M}\end{array}$ & $\begin{array}{l}39 \\
53\end{array}$ & $\begin{array}{ll}5 & . . \\
2 & \end{array}$ & $\begin{array}{l}150 / 120 \\
124 / 80\end{array}$ & $\begin{array}{l}\text { Angina. } \\
\text { Negative. }\end{array}$ & 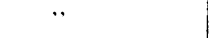 & $\begin{array}{l}170 \\
159 / 169\end{array}$ & Negative. \\
\hline 22 & M & 46 & $\begin{array}{ll}7 & \ddot{\prime}\end{array}$ & $120 / 80$ & Negative. & $\begin{array}{l}\text { Slight notching } P \\
\text { waves all leads. }\end{array}$ & 150 & ", \\
\hline $\begin{array}{l}23 \\
24\end{array}$ & $\begin{array}{l}\mathrm{M} \\
\mathbf{M}\end{array}$ & $\begin{array}{l}50 \\
49\end{array}$ & $\begin{aligned} & 7 \text { weeks } \\
& 18 \text { vears }\end{aligned}$ & $\begin{array}{l}120 / 84 \\
120 / 90\end{array}$ & 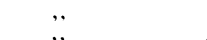 & & & Albumin : \\
\hline 25 & M & 66 & $10 \quad \cdots$ & $190 / 94$ & $\begin{array}{l}\text { Angina; left ven- } \\
\text { tricle enlarged. }\end{array}$ & & $193 / 156$ & $\begin{array}{l}\text { Aloumin, } \\
\text { Negative. }\end{array}$ \\
\hline 26 & $M$ & 45 & 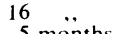 & $140 / 90$ & Systolic (apex). & & $155 / 162$ & " \\
\hline $\begin{array}{l}27 \\
28\end{array}$ & $\begin{array}{l}M \\
M\end{array}$ & $\begin{array}{l}47 \\
56\end{array}$ & $\begin{array}{l}5 \text { months } \\
27 \text { years }\end{array}$ & $\begin{array}{l}130 / 90 \\
120 / 70\end{array}$ & $\begin{array}{l}\text { Negative. } \\
\text { Angina. }\end{array}$ & & $\begin{array}{l}180 / 152 \\
180 / 155\end{array}$ & , \\
\hline 29 & M & 58 & $3 \quad$ & $126 / 78$ & Negative. & $\begin{array}{l}\text { Q.R.S. slurring all } \\
\text { leads. }\end{array}$ & & $\ddot{~ ", ~}$ \\
\hline 30 & $\mathbf{M}$ & 31 & 3 months & $136 / 76$ & $\begin{array}{l}\text { Soft systolic mur- } \\
\text { mur, praecor- } \\
\text { dium. }\end{array}$ & $\begin{array}{l}\text { Slurring Q.R.S. all } \\
\text { leads. }\end{array}$ & & " \\
\hline 31 & $M$ & 50 & 20 years & $140 / 80$ & Negative. & & $155 / 161$ & . \\
\hline 32 & $\mathbf{M}$ & 59 & 1 year & $140 / 80$ & $\begin{array}{l}\text { Loud apical sys- } \\
\text { tolic murmur. }\end{array}$ & & $150 / 147$ & $\because$ \\
\hline $\begin{array}{l}33 \\
34\end{array}$ & $\begin{array}{l}\mathbf{M} \\
\mathbf{M}\end{array}$ & $\begin{array}{l}48 \\
62\end{array}$ & $\begin{array}{l}1 \text { month } \\
27 \text { years }\end{array}$ & $\begin{array}{l}150 / 80 \\
130 / 78\end{array}$ & $\begin{array}{l}\text { Negative. } \\
\quad .\end{array}$ & $\begin{array}{l}\text { Slurring Q.R.S. all } \\
\text { leads. }\end{array}$ & & $\ddot{.}$ \\
\hline $\begin{array}{l}35 \\
36\end{array}$ & $M$ & 48 & 9 months & $104 / 60$ & ". & & & Trelbumin \\
\hline 36 & M & 48 & 17 years & $135 / 90$ & C1. & $\begin{array}{l}\text { Notched } P \text { waves, } \\
\text { and slurring } \\
\text { Q.R.S. all leads. }\end{array}$ & & I race albumin. \\
\hline 37 & M & 62 & $4 \quad$. & $160 / 86$ & $\begin{array}{l}\text { Slight enlarged left } \\
\text { ventricle. }\end{array}$ & & & $\begin{array}{l}\text { Trace albumin; rare } \\
\text { hyalin cast. }\end{array}$ \\
\hline $\begin{array}{l}38 \\
39\end{array}$ & $\begin{array}{l}\mathbf{M} \\
\mathbf{M}\end{array}$ & $\begin{array}{l}55 \\
72\end{array}$ & $\begin{array}{r}2 \\
40\end{array}$ & $\begin{array}{l}150 / 100 \\
145 / 70\end{array}$ & Negative. & & & Negative. \\
\hline 40 & $\mathbf{M}$ & 43 & $\begin{aligned} 40 & . \\
2 & . \cdot\end{aligned}$ & $\begin{array}{c}145 / 100 \\
\text { (112/70 four years }\end{array}$ & $\ddot{*}$ & & & ," \\
\hline 41 & M & 69 & 2 months & $\begin{array}{c}\text { before onset.) } \\
180 / 100\end{array}$ & $\begin{array}{l}\text { Old infarct by } \\
\text { E.C.G. }\end{array}$ & & & $\cdot$ \\
\hline $\begin{array}{l}42 \\
43\end{array}$ & $\begin{array}{l}M \\
M\end{array}$ & $\begin{array}{l}49 \\
49\end{array}$ & $\begin{array}{r}2 \text { years } \\
18\end{array}$ & $\begin{array}{l}120 / 80 \\
120 / 90\end{array}$ & Negative. & & $174 / 162$ & , \\
\hline 44 & $\mathbf{M}$ & 49 & 3 weeks & $150 / 86$ & $\ddot{\prime}$ & & $\begin{array}{l}\text { Over- } \\
\text { weight. }\end{array}$ & $\ddot{~}$ \\
\hline 45 & $\mathbf{M}$ & 57 & 1 week & $140 / 80$ & , & $\begin{array}{l}\text { Slight slurring all } \\
\text { leads. }\end{array}$ & & \\
\hline 46 & $\mathbf{M}$ & 50 & 20 years & $\begin{array}{l}140 / 80 \\
(2 \text { years later } 160 / \\
106\end{array}$ & $\begin{array}{l}\text { Enlarged left ven- } \\
\text { tricle. }\end{array}$ & $\begin{array}{l}\text { Slight slurring all } \\
\text { leads. }\end{array}$ & & , \\
\hline
\end{tabular}

the second, aged 66 , for ten years, and the third, aged 56 , for twenty-seven years.

\section{Drugs in the Treatment of Gout}

There is little question that the prevention of acute attacks of arthritis is desirable. More is gained by this prophylaxis than the mere comfort of the patient, for chronic gouty arthritis with its destructive joint changes is thus prevented. The use of cinchophen, a hepato-toxic agent, would be profitably replaced by colchicine if found equally efficacious. The following safe and effective method has been employed with this end in view. The patient with an acute attack of gouty arthritis is placed on absolute bed rest. A low-fat, highcarbohydrate, restricted nucleo-protein diet is prescribed. All alcoholic beverages and all activities which place an unusual stress or strain upon the joint are prohibited. Colchicine-1/100 grain $(0.65 \mathrm{mgm}$.) - is given by mouth every hour until one of two things occurs. Either the patient develops diarrhoea or the acute joint symptoms subside, usually after taking twelve to eighteen tablets. 
The drug is then stopped for twenty-four hours and resumed later, on a reduced dosage of one tablet three times daily. The exact amount required to produce either the diarrhoea or the relief of joint symptoms is noted in the record, an observation of use if the patient subsequently appears to be developing an acute attack of arthritis, or if the blood uric acid rises to a level above $5 \mathrm{mg}$. per $100 \mathrm{c.cm}$. The patient should then be given colchicine just short of the dosage that formerly produced gastro-intestinal symptoms. Blood uric acid estimations are necessary at three to four weekly intervals in order to carry out this control. If the blood uric acid is under $5 \mathrm{mg}$. per $100 \mathrm{c.cm}$. and no joint symptoms are present, it is reasonably safe to continue one tablet of colchicine three times daily until the next examination.

Many patients who were nearing the so-called gouty arthritic stage, in which they were getting attacks as frequently as every six weeks, have remained symptom free and in constant employment under this management. Colchicine is a much safer drug than cinchophen. No unusual findings in the electrocardiograph, and no change in the blood pressure or the pulse were observed in a patient who had received as many as twenty-two $1 / 100$-grain tablets of colchicine at hourly intervals. Occasionally, however, one finds a gouty patient who does better with large doses of salicylates than with colchicine. These people may be placed on 60 to 80 grains of salicylates for three days of the week and colchicine 1/100 grain three times daily for the remainder of the week. This regimen is also useful for those who develop gastro-intestinal symptoms on larger doses of colchicine.

\section{Summary}

1. Forty-six cases of gout were studied. Fourteen had hypertension and the remaining 32 had normal blood pressure.

2. Evidence is presented that gout is not an aetiological factor in hypertension.

3. There is little evidence that gout causes renal irritation. There is no evidence in our series to support the contention that gout leads to renal lithiasis. Only one case of renal lithiasis was present in our series, and this patient had a stone seven years before the onset of gouty symptoms and has had no recurrent stones since the removal of this one.

4. There is no evidence in this series to support the contention that gout parallels angina pectoris.

5. The value of colchicine as a prophylactic against acute attacks of gout is discussed.
TABle 2.-Gout Associated with Normal Blood Pressure

\begin{tabular}{|c|c|c|c|c|}
\hline Case & Age & $\begin{array}{l}\text { Duration of } \\
\text { disease }\end{array}$ & Sex & $\begin{array}{c}\text { Blood } \\
\text { pressure }\end{array}$ \\
\hline $\begin{array}{r}1 \\
2 \\
3 \\
4 \\
5 \\
6 \\
7 \\
8 \\
9 \\
10 \\
11 \\
12 \\
13 \\
14 \\
15 \\
16 \\
17 \\
18 \\
19 \\
20 \\
21 \\
22 \\
23 \\
24 \\
25 \\
26 \\
27 \\
28 \\
29 \\
30 \\
31\end{array}$ & $\begin{array}{l}57 \\
60 \\
53 \\
68 \\
56 \\
42 \\
51 \\
41 \\
45 \\
39 \\
40 \\
45 \\
72 \\
49 \\
49 \\
57 \\
55 \\
53 \\
46 \\
50 \\
49 \\
45 \\
47 \\
56 \\
58 \\
31 \\
62 \\
48 \\
48 \\
50 \\
59\end{array}$ & 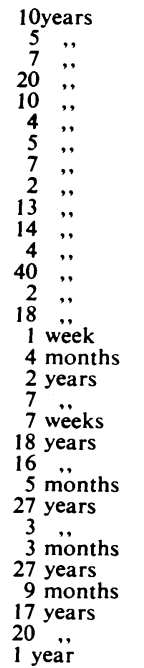 & $\begin{array}{l}\mathbf{M} \\
\mathbf{M} \\
\mathbf{M} \\
\mathbf{M} \\
\mathbf{M} \\
\mathbf{M} \\
\mathbf{M} \\
\mathbf{M} \\
\mathbf{M} \\
\mathbf{M} \\
\mathbf{M} \\
\mathbf{M} \\
\mathbf{M} \\
\mathbf{M} \\
\mathbf{M} \\
\mathbf{M} \\
\mathbf{M} \\
\mathbf{M} \\
\mathbf{M} \\
\mathbf{M} \\
\mathbf{M} \\
\mathbf{M} \\
\mathbf{M} \\
\mathbf{M} \\
\mathbf{M} \\
\mathbf{M} \\
\mathbf{M}\end{array}$ & $\begin{array}{l}140 / 80 \\
124 / 90 \\
120 / 80 \\
120 / 80 \\
114 / 80 \\
120 / 80 \\
120 / 80 \\
136 / 90 \\
140 / 80 \\
120 / 72 \\
120 / 80 \\
120 / 80 \\
145 / 70 \\
120 / 80 \\
120990 \\
140 / 80 \\
120 / 80 \\
12480 \\
120 / 80 \\
120 / 84 \\
120 / 90 \\
140 / 90 \\
130 / 90 \\
120 / 70 \\
126 / 78 \\
136 / 76 \\
130 / 78 \\
104 / 60 \\
13590 \\
140 / 80 \\
140 / 80\end{array}$ \\
\hline
\end{tabular}

TABLE 3.-Gout Associated with Hypertension

\begin{tabular}{|c|c|c|c|c|}
\hline Case & Age & $\begin{array}{l}\text { Duration of } \\
\text { disease }\end{array}$ & Sex & $\underset{\text { pressure }}{\text { Blood }}$ \\
\hline $\begin{array}{r}1 \\
2 \\
3 \\
4 \\
5 \\
6 \\
7 \\
8 \\
9 \\
10 \\
11 \\
12 \\
13 \\
14 \\
15\end{array}$ & $\begin{array}{l}51 \\
47 \\
39 \\
61 \\
53 \\
62 \\
55 \\
43 \\
69 \\
49 \\
50 \\
47 \\
39 \\
66 \\
48\end{array}$ & $\begin{array}{c}24 \text { years } \\
7 \text { months } \\
3 \text { years } \\
10 \text { "̈ } \\
6 \text { weeks } \\
4 \text { years } \\
2 \text { ", } \\
2 \text { \#̈ } \\
2 \text { months } \\
3 \text { weeks } \\
20 \text { years } \\
9 \text {, } \\
5 \text {, } \\
10 \text {, } \\
1 \text { month }\end{array}$ & $\begin{array}{l}\mathbf{M} \\
\mathbf{M} \\
\mathbf{M} \\
\mathbf{M} \\
\mathbf{F} \\
\mathbf{M} \\
\mathbf{M} \\
\mathbf{M} \\
\mathbf{M} \\
\mathbf{M} \\
\mathbf{M} \\
\mathbf{M} \\
\mathbf{M} \\
\mathbf{M} \\
\mathbf{M}\end{array}$ & $\begin{array}{l}160 / 80 \\
140 / 110 \\
140 / 100 \\
180 / 100 \\
170 / 100 \\
160 / 86 \\
150 / 100 \\
145 / 100 \\
180 / 100 \\
150 / 86 \\
160 / 106 \\
190 / 110 \\
150 / 120 \\
190 / 94 \\
150 / 80\end{array}$ \\
\hline
\end{tabular}

\section{REFERENCES}

Buckley, C. W. (1937). British Encvclopaedia of Medical Practice, 6,45 .

(1938). Arthritis, Fibrositis, and Gout, Lewis, London, p. 130. R. L. (1943). A Textbook of Medicine, 6th Edition, Philadelphia, W. B. Saunders Co.

Garcin, R. (1939). Progrès Med. 67, 588-592.

Levine, S. A. (1940). Clinical Heart Disease, 2nd Edition, Philadelphia, W. B. Saunders Co.

Lockie, L. M. (1942). "Diagnosis of 'Initial' Attack of Gouty Arthritis," Clinics, 1, 571.

, and Hubbard, R. S. (1935). J. Amer. Med. Ass., 104, 2072.

Master, M., Jaffe, H. L., Dack, S., and Silver, N. (1943). Amer. Heart J., 26, 92.

Meakin, J. (1939). Practice of Medicine, 2nd Edition, St. Louis, C. V. Mosby Co.

Osler, W., and McCrae (1925). Osler's Principles and Practice of Medicine, 10th Edition, New York, E. Appleton and Co.

Rosenbloom, J. (1918). J. Amer. Med. Ass., 70, 2000.

Stotzer, E. (1939). Schweiz med. Wschr. 69, 29.

Sydenham, Thomas (1683). "A Treatise of the Gout and Dropsy," published in London May 21, 1683, and quoted in Medical Classics, 4, 354, 1940. 